\title{
An Inductive Approach to Provable Anonymity
}

\author{
Yongjian Li \\ The State Key Laboratory of Computer Sciences \\ Institute of Software \\ Chinese Academy of Sciences
}

\author{
Jun Pang \\ Computer Science and Communications \\ University of Luxembourg
}

\begin{abstract}
We formalise in a theorem prover the notion of provable anonymity proposed by Garcia et al. Our formalization relies on inductive definitions of message distinguish ability and observational equivalence over observed traces by the intruder. Our theory differs from its original proposal which essentially boils down to the existence of a reinterpretation function. We build our theory in Isabelle/HOL to have a mechanical framework for the analysis of anonymity protocols. Its feasibility is illustrated through the onion routing protocol.
\end{abstract}

\section{INTRODUCTION}

With the rapid growth of the Internet community and the rapid advances in technology over the past decades, people are getting used to carry out their daily activities through networked distributed systems providing electronic services to users. In these systems, people become more and more concerned about their privacy and how their personal information has been used. Typically, anonymity is a desired property of such systems, referring to the ability of a user to own some data or take some actions without being tracked down. This property is essential in systems that might involve sensitive personal data, like electronic auctions, voting, anonymous broadcasts, file-sharing etc. For example, users want to keep anonymous when they visit a particular web site or post their political opinions on a public bulletin board.

Due to its subtle nature, anonymity has been the subject of many theoretical studies and formal verification [1], [2], [3], [4], [5]. The proposed definitions aim to capture different aspects of anonymity (either possibilisticor probabilisticand formal verification treats systems in different application domains, such as electronic voting systems electronic cash protocols file sharing. However, automatic approaches to the formal verification of anonymity have mostly focused on the model checking approach on systems with fixed configurations [1], while theorem proving is a more suitable approach when dealing with general systems of infinite state spaces. We address this situation by investigating the possibility of using a powerful general-purpose theorem prover, Isabelle/HOL [6], to semi-automatically verify anonymity properties.

We start by formalising the notion of provable anonymity proposed by Garcia et al. [2]. Their key idea is to define observational equivalence between protocol traces. Two traces are to be considered equivalent if an intruder cannot distinguish them, i.e., he cannot find any meaningful difference. The distinguishing ability of the intruder is formalised as the ability to distinguish two messages, which is in turn based on message structures and relations between random looking messages. Central to their framework is the reinterpretation function proposed by Garcia et al. [2]. Proving two traces equivalent essentially boils down to the existence of such a reinterpretation function. Within their framework, Garcia et al. also define epistemic operators and use them to express information hiding properties like sender anonymity and unlinkability.

Our contribution: Our formalization of observational equivalence between traces relies on a definition of message distinguishability. Observational equivalence of traces is in the center of the epistemic framework - an agent knows a fact of a certain trace if that fact is true in all traces that are observationally equivalent to that trace. We build our theory in Isabelle/HOL [6] to have a mechanical framework for the analysis of anonymity protocols. We illustrate the feasibility of the mechanical framework through the onion routing protocol [7]. We inductively define the semantics of an onion routing protocol as a set of traces, and the relaying mechanism of the protocol is formally defined as a set of inductive rules. Furthermore, we formally prove that the protocol realizes anonymity properties such as sender anonymity and unlinkability under some circumstance by providing a method to construct an observationally equivalent onion trace for a given trace. To the best of our knowledge, theory of anonymity has not been formalised in a theorem prover yet. Our work aims to to bridge this gap. All lemmas in the paper are proved semi-automatically in Isabelle/HOL. Proofs are mostly omitted for the sake of brevity.

\section{PRELIMINARIES}

\section{A. Agents, messages and events}

Agents send or receive messages. There are three kinds of agents: the server, the friendly agents, and the spy. Formally the type of agent is defined as follows:

$$
\text { agent }::=\text { Server } \mid \text { Friend } N \mid \text { Spy }
$$

We use bad to denote the set of intruders, which at least includes the agent Spy. If an agent $A$ is not in bad, then $A$ is honest.

The set of messages is defined using the following BNF notation:

$$
h::=\text { Agent } A \quad \mid \text { Nonce } N \quad \mid \text { Key } K
$$


anonymity with anonymity set $A S$ w.r.t a set of possible runs in the view of $B$ if it satisfies:

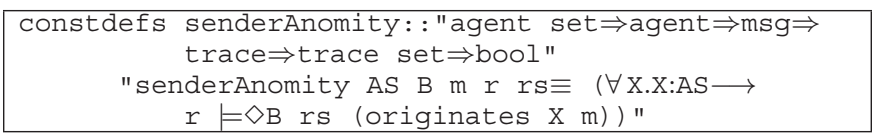

Here, $A S$ is the set of agents who are under consideration, and $r s$ is the set of all the traces which $B$ can observe. Intuitively, this definition means that each agent in $A S$ can originate $m$ in a trace of $r s$. Therefore, this means that $B$ cannot be sure of anyone who originates this message.

\section{B. Unlinkability}

We say that a trace $r$ provides unlinkability for user $A$ and a message $m$ w.r.t anonymity set $A S$ if

\begin{tabular}{|c|c|}
\hline $\begin{array}{r}\text { constdefs } \\
\text { "unl }\end{array}$ & 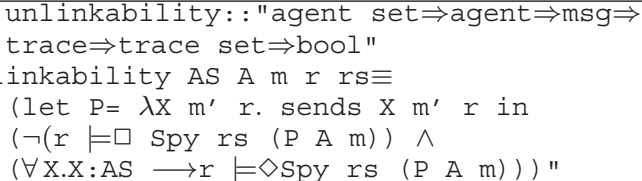 \\
\hline
\end{tabular}

where the left side of the conjunction means that the intruder is not certain that $A$ sent $m$, while the right side means that every other user could have sent $m$.

\section{Case Study: Onion Routing Protocol}

\section{A. Modeling the protocol}

In our work, we model a simplified onion routing protocol system, composed of a user set $A S$ and a router $M$, with $M \notin A S$. We also assume that each agent can send a message before the router $M$ launch a batch of forwarding process, and the router does not accept any message when it is forwarding messages.

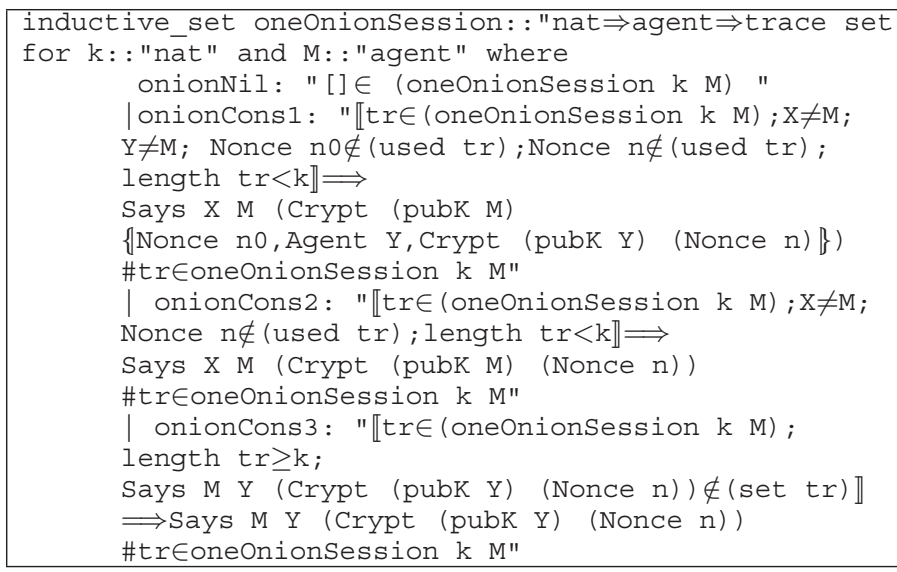

In this definition, there are four induction rules. Rule $\mathrm{NiI}$ specifies an empty trace. The other rules specify trace's extension with protocol steps. The ideas behind the other induction rules are illustrated as follows. More precisely,

- If the length of the current trace is less than $k$, namely, $M$ is still in a receiving status, $X$ (or $Y$ ) and $M$ are distinct, and both $n 0$ and $n$ are fresh, then we can add an event Says $X M\left\{\right.$ Nonce $n_{0}$, Agent $Y,\{$ Nonce $n\}$ pubK $\left.Y\right\}$ pubK $M$
This step means that $X$ sends a message to $M$ which will be peeled and forwarded to $Y$ by $M$.

- If the length of the current trace is less than $k, X$ and $M$ are distinct, and $n$ is fresh, then we can add an event Says $X M\{\text { Nonce } N\}_{\text {pubk } M}$. This means that $X$ sends a dummy message to $M$ which will be simply discarded later.

- If the length of the current trace is greater than or equal to $k$, namely, $M$ is in a forwarding status, a message $\left\{\text { Nonce } n_{0} \text {, Agent } Y,\{\text { Nonce } n\}_{\text {pubK } Y}\right\}_{\text {pubk } M}$ has been received by the router, but the peeled onion $\{\text { Nonce } n\}_{\text {pubK } Y}$ has not been forwarded, then we can add an event Says $M Y\{\text { Nonce } n\}_{\text {pubk } Y}$. This step means that the router $M$ forwards the peeled message to $Y$.

\section{B. Properties on protocol sessions}

As mentioned in a previous section, whether two traces are observationally equivalent for an agent depends on the knowledge of the agent after his observation of the two traces. Therefore, we need to discuss some properties on the knowledge of the intruder. They are secrecy properties, and some regularity on the correspondence of the events in one protocol session.

a) Secrecy properties.: If the router $M$ is honest, $B$ is also honest, and $B$ sends a message $\left\{\text { Nonce } n_{0} \text {, Agent } Y,\{\text { Nonce } n\}_{\text {pubK } Y}\right\}_{\text {pubK } M}$ to $M$, either $n_{0} \neq n$ or $Y \notin$ bad, then Nonce $n_{0}$ cannot be analyzed by the intruder.

\section{Lemma 1}

$\llbracket t r \in$ oneOnionSession $k M ; n_{0} \neq n \vee Y \notin$ bad; Says $B M\left\{\text { Nonce } n_{0} \text {, Agent } Y,\{\text { Nonce } n\}_{\text {pubK } Y}\right\}_{\text {pubK } M} \in$ tr; $M \notin \mathrm{bad} ; B \notin \mathrm{bad} \rrbracket \Longrightarrow$ Nonce $n_{0} \notin$ analz (spies evs)

Provided that both $M$ and $B$ are honest, and $B$ sends a dummy message $\left\{\text { Nonce } n_{0}\right\}_{\text {pubK } M}$ to $M$, then Nonce $n_{0}$ cannot be analyzed by the intruder.

\section{Lemma 2}

$\llbracket t r \in$ oneOnionSession $k M$; Says $B M\left\{\text { Nonce } n_{0}\right\}_{\text {pubk } M} \in$ $\operatorname{tr} ; M \notin \mathrm{bad} ; B \notin \mathrm{bad} \rrbracket \Longrightarrow$ Nonce $n_{0} \notin$ analz (spies evs)

b) Correspondence properties.: The following lemma is about the correspondence of two events in a trace $t r$. If the router $M$ forwards a message $\{\text { Nonce } n\}_{\text {pubk } Y}$, then there must exist an agent $A$ who has sent a message for some nonce $n_{0}\left\{\text { Nonce } n_{0}, \text { Agent } Y,\{\text { Nonce } n\}_{\text {pubK } Y}\right\}_{\text {pubK } M}$.

\section{Lemma 3}

$\llbracket t r \in$ oneOnionSession $k M$;

$m a^{\prime}=\{\text { Nonce } n\}_{\text {pubK } Y} ;$ Says $M B m a^{\prime} \in$ set $t r ; \rrbracket \Longrightarrow$ $\exists n_{0} A$.Says $\left.A M\left\{\text { Nonce } n_{0} \text {, Agent } Y,\{\text { Nonce } n\}\right\}_{\text {pubk } Y}\right\}_{\text {pubK } M} \in$ set $\operatorname{tr}$

If $\{\text { Nonce } n\}_{\text {pubK } Y}$ is a submessage of a message which $A$ sends to the router $M$, then $\{\text { Nonce } n\}_{\text {pubK } Y}$ is originated by $A$. 


\section{Lemma 4}

$\llbracket t r \in$ oneOnionSession $k M$;

$m a^{\prime}=\{\text { Nonce } n\}_{\text {pubk } Y}$; Says $A M m a \in$ set $\operatorname{tr} ; m a^{\prime} \sqsubset$ $\mathrm{ma} \Longrightarrow$ originates $A m a^{\prime} \mathrm{tr}$

For a trace $t r \in$ oneOnionSession $k M$, an agent $A$ sends the router $M$ a message $m$, then $A$ is not the router $M$.

\section{Lemma 5}

$\llbracket t r \in$ oneOnionSession $k M$; Says $A M m \in$ set $t r \rrbracket \Longrightarrow$ $A \neq M$

c) Uniqueness properties.: Since an agent is required to originate fresh nonces when he sends a message to the router, therefore if two events where agents send a message to the router $M$, either two events are exactly the same, or nonces used in the two events are disjoint.

\section{Lemma 6}

$\llbracket t r \in$ oneOnionSession $k M$;

Says $X M m a$; Says $Y M m b \rrbracket \Longrightarrow(X=Y \wedge m a=m b) \vee$ (noncesOf $m a) \cap($ noncesOf $m b)=\emptyset$

From Lemma 6, we can easily derive that once a nonce $n$ occurs in a message sent by an agent $X$, then another agent $Y$ cannot send a message containing the same nonce $n$.

\section{Lemma 7}

$\llbracket t r \in$ oneOnionSession $k M$; Says $X M m a$;

$X \neq Y$; Nonce $n \sqsubset m a \rrbracket \Longrightarrow \neg$ sends $Y$ (Nonce $n$ ) $t r$

The message of each event in a trace of the protocol is unique, namely two messages in two events in this trace are different.

\section{Lemma 8}

$\llbracket t r \in$ oneOnionSession $k \quad M \rrbracket \Longrightarrow$ map msgPart $\operatorname{tr} \in$ mutualDiffL

(zip (map msgPart $t r$ ) $L$ ) must be single_valued if $t r$ is in a trace of the onion routing protocol.

Lemma $9 \llbracket t r \in$ oneOnionSession $k M \rrbracket$ $\Longrightarrow$ single_valued (zip (map msgPart $t r$ ) $L$ )

\section{Traces swapping two messages}

By definition of sender anonymity, the proof strategy of such property is roughly as follows: fix an agent $X$, we need to prove the existence of an observationally equivalent trace $t r^{\prime}$ w.r.t. a given trace $t r$, where both $t r$ and $t r^{\prime}$ are some protocol sessions. Obviously, this means a construction of an observationally equivalent trace $t r^{\prime}$. In this section, we discuss this construction method in details.

We define a function swap ma $m b t r$, which returns another trace $t r^{\prime}$ satisfying that the sender and receiver of any event $t r_{i}^{\prime}$ is the same as those in $t r_{i}$, but the sent message of $t r_{i}^{\prime}$ is

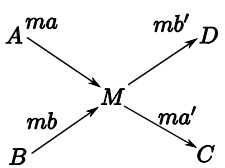

(1)

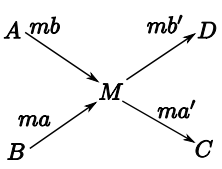

(2)

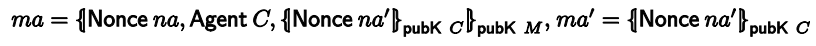
$m b=\left\{\text { Nonce } n b \text {, Agent } D,\left\{\text { Nonce } n b^{\prime}\right\}_{\text {pubK } D}\right\}_{\text {pubK } M}, m b^{\prime}=\left\{\text { Nonce } n b^{\prime}\right\}_{\text {pubK } D}$

Fig. 1. An illustration of function swap.

swapped as $m b$ if that of $t r_{i}$ is $m a$, and as $m a$ if that of $t r_{i}$ is $m b$, otherwise it is kept the same as that of $t r_{i}$.

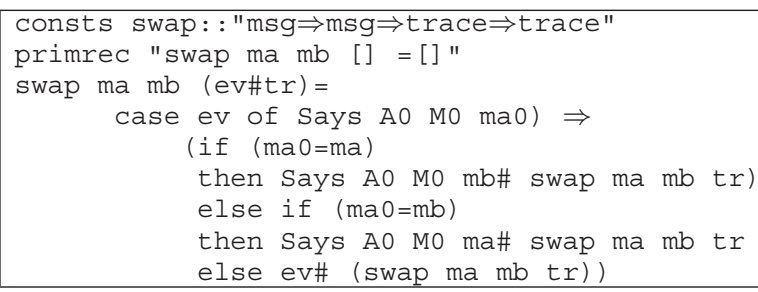

For a trace $t r$ of the onion routing protocol, Fig. 1 illustrates the correspondence between $\operatorname{tr}$ and the function swap ma mb tr. In session 1, agent $A(B)$ communicates with $C(D)$, while agent $A(B)$ communicates with $D(C)$ in session 2. The correspondence between $t r$ and swap ma $m b$ tr is formalised as the following lemma.

Lemma 10 Let tr be a trace.

1) $\llbracket\left(m_{1}, m_{2}\right) \in$ set (zip (map msgPart $\left.t r\right)$ $($ map msgPart (swap ma $m b$ tr) $)) \rrbracket \Longrightarrow m_{1}=m_{2} \vee$ $\left(m_{1}, m_{2}\right)=(m a, m b) \vee\left(m_{1}, m_{2}\right)=(m b, m a)$

2) sendRecvMatchL $t r$ (swap ma $m b t r$ )

3) length ( swap $m a m b t r)=$ length $t r$

4) swap $m a m b t r=$ swap $m b m a t r$

5) $\llbracket($ Says $X M m a \in$ set $t r) \rrbracket$ $\Longrightarrow$ Says $X M m b \in$ set (swap $m a m b t r)$ )

6) $\llbracket($ Says $X M m b \in$ set $t r) \rrbracket$ $\Longrightarrow$ Says $X M m a \in$ set (swap ma mb tr))

7) $\llbracket m \neq m a ; m \neq m b$; (Says $X M m) \in$ set $t r \rrbracket$ $\Longrightarrow($ Says $X M m \in$ set (swap ma $m b$ tr $)$ )

8) $\llbracket m \neq m a ; m \neq m b$; (Says $X M m) \notin$ set $t r \rrbracket$ $\Longrightarrow($ Says $X M m \notin$ set (swap ma $m b t r)$ )

9) \Says $A M m a \in t r$; Says $B M m b \in t r ; A \neq$ Spy; $B \neq \mathrm{Spy} \rrbracket$

$\Longrightarrow$ knows Spy $t r=$ knows Spy (swap $m a m b t r$ )

Based on the lemma 10, we can conclude an important result: for a trace $t r \in$ oneOnionSession $k M$, both $m a$ and $m b$ are sent to the router $M$ by some agents in $t r$, then swap ma $m b t r$ is still in oneOnionSession $k M$.

\section{Theorem 1}

$\llbracket t r \in$ oneOnionSession $k$ M; Says $A \quad M \quad m a \in$ $t r$; Says $B \quad M m b \in t r \rrbracket \Longrightarrow$ swap ma mb tr $\in$ oneOnionSession $k M$ 
If $\left.m a=\left\{\text { Nonce } n_{0} \text {, Agent } Y \text {, \{Nonce } n\right\}_{\text {pubK } Y}\right\}_{\text {pubK } M}$, $m a$ is sent to the router $M$ by an honest agent $A$, and $m b$ is also sent to the router $M$ by an honest agent $B$, then $t r$ is observationally equivalent to swap $m a m b t r$ in the view of the Spy.

\section{Lemma 11}

$\llbracket t r \in$ oneOnionSession $k M$;

$m a=\left\{\text { Nonce } n_{0} \text {, Agent } Y,\{\text { Nonce } n\}_{\text {pubk } Y}\right\}_{\text {pubk } M}$;

Says $A M m a \in$ set $t r$; Says $B M m b \in$ set $t r$;

$A \notin$ bad; $M \notin$ bad; $B \notin$ bad; $n_{0} \neq n \vee Y \notin$ bad】

$\Longrightarrow$ obsEquiv Spy $\operatorname{tr}$ (swap ma mb tr)

\section{Proving anonymity properties}

Message $m a^{\prime}$ is forwarded to $B$ by the router $M$, and is originated by some honest agent, and the nonce $n$ satisfies a constraint cond $\operatorname{tr} M n$, which will be explained in details later, then spy cannot be sure of the honest agent who originates $m a^{\prime}$. Namely, the sender anonymity holds for the intruder w.r.t. the honest agents who send messages to $M$ in the session modelled by $t r$.

\section{Lemma 12}

$\llbracket t r \in$ oneOnionSession $k M$; $m a^{\prime}=\{\text { Nonce } n\}_{\text {pubk } Y}$;

Says $M B m a^{\prime} \in$ set $t r$; regularOrig $m a^{\prime} t r$;

$M \notin$ bad; cond $\operatorname{tr} M \rrbracket \Longrightarrow$

senderAnomity (senders $\operatorname{tr} M-$ bad)

Spy $m a^{\prime} \operatorname{tr}$ (oneOnionSession $k M$ ), where senders $\operatorname{tr} M \equiv$ $\{A . \exists m$.Says $A M m \in$ set $t r\}$, and cond $\operatorname{tr} M \equiv$ $\forall A n_{0} n Y$.Says $A M\left\{\right.$ Nonce $n_{0}$, Agent $\left.Y,\{\text { Nonce } n\}_{\text {pubk } Y}\right\}$ pubk $M \in$ set $t r \longrightarrow\left(Y \notin \operatorname{bad} \vee n_{0} \neq n\right)$

The premise cond $\operatorname{tr} M n$ says that if a nonce $n$ is originated in a message $\left.\left\{\text { Nonce } n_{0} \text {, Agent } Y \text {, \{Nonce } n\right\}_{\text {pubK } Y}\right\}_{\text {pubK } M}$ in the trace $t r$, then either $Y \notin$ bad or $n_{0} \neq n$, this guarantees the secrecy of $n$.

The last result is about the linkability of a sender $A$ and a peeled onion ma. Suppose that an honest agent $A$ sends a message $m$ to the router $M$, and an agent $B$ receives a message $m a$ from $M$, the intruder cannot link the message $m a$ ' with the agent $A$ provided that there exists at least one agent $X$ who is not $A$ and sends a message to $M$.

\section{Lemma 13}

$\llbracket t r \in$ oneOnionSession $k M$;

$m a^{\prime}=\{\text { Nonce } n\}_{\text {pubk } Y}$;

Says $M B m a^{\prime} \in$ set $t r$; regularOrig $m a^{\prime}$ tr;

Says $A M m^{\prime} \in$ set $t r ; A \notin$ bad; $M \notin$ bad;

$\exists X, m x$. Says $X M m x \in$ set $\operatorname{tr} \wedge X \neq A \wedge X \notin$

bad; cond $\operatorname{tr} M n \rrbracket$

$\Longrightarrow$ let $A S=$ senders $\operatorname{tr} M-$ bad in

unlinkability $A S A m$ (oneOnionSession $k M$ )

\section{ViI. CONCLUSion AND Future Work}

In this work, we formalise the notion of provable anonymity in the theorem prover Isabelle/HOL. First we propose an inductive definition of message distinguishability based on the observer's knowledge, then define the message equivalence as the negation of message distinguishability. Next, we define observational equivalence of two traces using the message equivalence, and define the semantics of anonymity properties in an epistemic logical framework. In the end, we inductively formalise the semantics of the onion routing protocols, and formally prove that sender anonymity and unlikability hold for the protocol in Isabelle/HOL.

When we prove that properties such as sender anonymity hold for a trace under consideration, we need to consider the existence of another trace which is observationally equivalent to the given trace, but differs, for example, in the sender of some message. This is the essence of information hiding on the senders or the linkage between a message and its sender, which makes the analysis of anonymity different from analysis on secrecy and authentication. For secrecy and authentication, normally the focus is on individual traces. However, the observer decides whether two traces are observationally equivalent according to his knowledge obtained in the two traces, which usually boils down to the secrecy of some terms. Therefore, the induction proof method used in the analysis of secrecy properties can still be used here. This may be the relation between analysis on classical protocol properties on secrecy and that on anonymity properties.

In future, we will apply our framework to more case studies. We would also like to check whether our framework can be easily generalised to model different kinds of privacy and information hiding properties and to model protocols which allow more cryptographic primitives. Theoretically, we believe this inductive approach can be extended because only additional induction rules are required. In particular, it is interesting for us to find out whether the method of constructing an observationally equivalent trace using the swap function is general enough.

\section{REFERENCES}

[1] S. Schneider and A. Sidiropoulos, "CSP and anonymity," in Proc. 4th European Symposium on Research in Computer Security, ser. LNCS, vol. 1146. Springer, 1996, pp. 198-218.

[2] F. D. Garcia, I. Hasuo, W. Pieters, and P. van Rossum, "Provable anonymity," in Proc. 3rd Workshop on Formal Methods in Security Engineering. ACM, 2005, pp. 63-72.

[3] A. Pfitzmann and M. Hansen, "A terminology for talking about privacy by data minimization: Anonymity, unlinkability, undetectability, unobservability, pseudonymity, and identity management," April 2010.

[4] S. Mauw, J. Verschuren, and E. P. d. Vink, "A formalization of anonymity and onion routing," in Proc. 9th European Symposium on Research in Computer Security, ser. LNCS, vol. 3193. Springer, 2004, pp. 109-124.

[5] S. Kremer and M. Ryan, "Analysis of an electronic voting protocol in the applied pi-calculus," in Proc. 14th European Symposium on Programming, ser. LNCS, vol. 3444. Springer, 2005, pp. 186-200.

[6] T. Nipkow, L. C. Paulson, and M. Wenzel, Isabelle/HOL - A Proof Assistant for Higher-Order Logic, ser. LNCS. Springer, 2002, vol. 2283.

[7] P. F. Syverson, D. M. Goldschlag, and M. G. Reed, "Anonymous connections and onion routing," in Proc. 18th IEEE Symposium on Security and Privacy. IEEE, 1997, pp. 44-54.

[8] L. C. Paulson, "The inductive approach to verifying cryptographic protocols," Journal of Computer Security, vol. 6, no. 1-2, pp. 85-128, 1998.

[9] D. Dolev and A. C.-C. Yao, "On the security of public key protocols," IEEE Transactions on Information Theory, vol. 29, no. 2, pp. 198-207, 1983. 